\title{
Automated language essay scoring systems: A Literature Review
}

\author{
Mohamed Abdellatif Hussein ${ }^{\text {Corresp., } 1}$, Hesham Ahmed Hassan ${ }^{2}$, Mohamed Nassef $^{2}$ \\ 1 Information and Operations, National Center for Examination and Educational Evaluation, Cairo, Egypt \\ 2 Faculty of Computers and Information, Computer Science Department, Cairo University, Cairo, Egypt \\ Corresponding Author: Mohamed Abdellatif Hussein \\ Email address: teeefa@nceee.edu.eg
}

Background.Writing composition is a significant factor for measuring test-takers' ability in any language exam. However, the assessment (scoring) of these writing compositions or essays is a very challenging process in terms of reliability and time. The need for objective and quick scores has raised the need for a computer system that can automatically grade essay questions targeting specific prompts. Automated Essay Scoring (AES) systems are used to overcome the challenges of scoring writing tasks by using Natural Language Processing (NLP) and machine learning techniques. The purpose of this paper is to review the literature for the AES systems used for grading the essay questions. Methodology.We have reviewed the existing literature using Google Scholar, EBSCO and ERIC to search for the terms "AES", "Automated Essay Scoring", "Automated Essay Grading", or "Automatic Essay" for essays written in English language. Two categories have been identified: handcrafted features and automatically featured AES systems. The systems of the former category are closely bonded to the quality of the designed features. On the other hand, the systems of the latter category are based on the automatic learning of the features and relations between an essay and its score without any handcrafted features. We reviewed the systems of the two categories in terms of system primary focus, technique(s) used in the system, the need for training data, instructional application (feedback system), and the correlation between e-scores and human scores. The paper includes three main sections. First, we present a structured literature review of the available Handcrafted Features AES systems. Second, we present a structured literature review of the available Automatic Featuring AES systems. Finally, we draw a set of discussions and conclusions.Results.AES models have been found to utilize a broad range of manually-tuned shallow and deep linguistic features. AES systems have many strengths in reducing labor-intensive marking activities, ensuring a consistent application of scoring criteria, and ensuring the objectivity of scoring. Although many techniques have been implemented to improve the AES systems, three primary challenges have been identified. The challenges are lacking of the 
sense of the rater as a person, the potential that the systems can be deceived into giving a lower or higher score to an essay than it deserves, and the limited ability to assess the creativity of the ideas and propositions and evaluate their practicality. Many techniques have been used to address the first two challenges only. 


\section{Automated language essay scoring systems: A} 3 Literature Review

4

5

6 Mohamed Abdullatif Hussein ${ }^{1}$, Hesham Ahmed Hassan², Mohamed Nassef ${ }^{2}$

7

$8{ }^{1}$ Information and Operations, National Center for Examination and Educational Evaluation, 9 Cairo, Cairo, Egypt

$10{ }^{2}$ Computer Science, Faculty of Computers and Information, Cairo University, Cairo, Egypt 11

12 Corresponding Author:

13 Mohamed Hussein ${ }^{1}$

14 84E Hadayk Ahram, Haram, Giza, 12556, Egypt

15 Email address: teeefa@nceee.edu.eg 


\section{Abstract}

18 Background. Writing composition is a significant factor for measuring test-takers' ability in any

19

20

21

22

23

24

25

26

27

28

29

30

31

32

33

34

35

36

37

38

39

40

41

42

43

44

45

46

47

48

49

50

51

52

53

54

55

56 language exam. However, the assessment (scoring) of these writing compositions or essays is a very challenging process in terms of reliability and time. The need for objective and quick scores has raised the need for a computer system that can automatically grade essay questions targeting specific prompts. Automated Essay Scoring (AES) systems are used to overcome the challenges of scoring writing tasks by using Natural Language Processing (NLP) and machine learning techniques. The purpose of this paper is to review the literature for the AES systems used for grading the essay questions. Methodology. We have reviewed the existing literature using Google Scholar, EBSCO and ERIC to search for the terms "AES", "Automated Essay Scoring", "Automated Essay Grading", or "Automatic Essay" for essays written in English language. Two categories have been identified: handcrafted features and automatically featured AES systems. The systems of the former category are closely bonded to the quality of the designed features. On the other hand, the systems of the latter category are based on the automatic learning of the features and relations between an essay and its score without any handcrafted features. We reviewed the systems of the two categories in terms of system primary focus, technique(s) used in the system, the need for training data, instructional application (feedback system), and the correlation between e-scores and human scores. The paper includes three main sections. First, we present a structured literature review of the available Handcrafted Features AES systems.

Second, we present a structured literature review of the available Automatic Featuring AES systems. Finally, we draw a set of discussions and conclusions. Results. AES models have been found to utilize a broad range of manually-tuned shallow and deep linguistic features. AES systems have many strengths in reducing labor-intensive marking activities, ensuring a consistent application of scoring criteria, and ensuring the objectivity of scoring. Although many techniques have been implemented to improve the AES systems, three primary challenges have been identified. The challenges are lacking of the sense of the rater as a person, the potential that the systems can be deceived into giving a lower or higher score to an essay than it deserves, and the limited ability to assess the creativity of the ideas and propositions and evaluate their practicality. Many techniques have been used to address the first two challenges only.

\section{Introduction}

Test items (questions) are usually classified into two types: selected-response (SR), and constructed-response (CR). The SR items, such as true/false, matching or multiple-choice, are much easier than the CR items in terms of objective scoring (Isaacs, 2013). SR questions are commonly used for gathering information about knowledge, facts, higher-order thinking, and problem-solving skills. However, considerable skill is required to develop test items that measure analysis, evaluation, and other higher cognitive skills (Stecher et al., 1997).

CR items, sometimes called open-ended, include two sub-types: restricted-response and extended-response items (Nitko \& Brookhart, 2007). Extended-response items, such as essays, problem-based examinations, and scenarios, are like restricted-response items, except that they extend the demands made on test-takers to include more complex situations, more difficult

Peer] Comput. Sci. reviewing PDF | (CS-2019:04:37187:1:0:NEW 13 Jun 2019) 
57 reasoning, and higher levels of understanding which are based on real-life situations requiring

58

59

60

61

62

63

64

65

66

67

68

69

70

71

72

73

74

75

76

77

78

79

80

81

82

83

84

85

86

87

88

89

90

91

92

93

94

95

test-takers to apply their knowledge and skills to new settings or situations (Isaacs, 2013). In language tests, test-takers are usually required to write an essay about a given topic. Humanraters score these essays based on specific scoring rubrics or schemes. It occurs that the score of an essay scored by different human-raters vary substantially because human scoring is subjective (Peng, Ke, \& Xu, 2012). As the process of human scoring takes much time, effort, and are not always as objective as required, there is a need for an automated essay scoring system that reduces cost, time and determines an accurate and reliable score.

Automated Essay Scoring (AES) systems usually utilize Natural Language Processing and machine learning techniques to automatically rate essays written for a target prompt (Dikli, 2006). Many AES systems have been developed over the past decades. They focus on automatically analyzing the quality of the composition and assigning a score to the text. Typically, AES models exploit a wide range of manually-tuned shallow and deep linguistic features (Farag, Yannakoudakis, \& Briscoe, 2018). Recent advances in the deep learning approach have shown that applying neural network approaches to AES systems has accomplished state-of-the-art results (Page, 2003; Valenti, Neri, \& Cucchiarelli, 2017) with the additional benefit of using features that are automatically learnt from the data.

\section{Survey methodology}

The purpose of this paper is to review the AES systems literature pertaining to scoring extendedresponse items in language writing exams. Using Google Scholar, EBSCO and ERIC, we searched the terms "AES", "Automated Essay Scoring”, “Automated Essay Grading”, or "Automatic Essay" for essays written in English language. AES systems which score objective or restricted-response items are excluded from the current research.

The most common models found for AES systems are based on Natural Language Processing (NLP), Bayesian text classification, Latent Semantic Analysis (LSA), or Neural Networks. We have categorized the reviewed AES systems into two main categories. The former is based on handcrafted discrete features bounded to specific domains. The latter is based on automatic feature extraction. For instance, Artificial Neural Network (ANN)-based approaches are capable of automatically inducing dense syntactic and semantic features from a text.

The literature of the two categories has been structurally reviewed and evaluated based on certain factors including: system primary focus, technique(s) used in the system, the need for training data, instructional application (feedback system), and the correlation between e-scores and human scores.

\section{Handcrafted Features AES Systems} Project Essay Grader ${ }^{\mathrm{TM}}$ (PEG)

Ellis Page developed the PEG in 1966. PEG is considered the earliest AES system that has been built in this field. It utilizes correlation coefficients to predict the intrinsic quality of the text. It uses the terms "trins" and "proxes" to assign a score. Whereas "trins" refers to intrinsic variables like diction, fluency, punctuation, and grammar, "proxes" refers to correlations between intrinsic 
96

97

98

99

100

101

102

103

104

105

106

107

108

109

110

111

112

113

114

115

116

117

118

119

120

121

122

123

124

125

126

127

128

129

130

131

132

133

134

135

variables such as average length of words in a text, and/or text length. (Dikli, 2006; Valenti et al., 2017).

The PEG uses a simple scoring methodology that consists of two stages. The former is the training stage and the latter is the scoring stage. PEG should be trained on a sample of essays from 100 to 400 essays, the output of the training stage is a set of coefficients ( $\beta$ weights) for the proxy variables from the regression equation. In the scoring stage, proxes are identified for each essay, and are inserted into the prediction equation. To end, a score is determined by estimating coefficients ( $\beta$ weights) from the training stage (Dikli, 2006).

Some issues have been marked as a criticism for the PEG such as disregarding the semantic side of essays, focusing on surface structures, and not working effectively in case of receiving student responses directly (which might ignore writing errors). PEG has a modified version released in 1990, which focuses on grammar checking with a correlation between human assessors and the system ( $r=0.87$ ) (Dikli, 2006; Page, 1994; Refaat, Ewees, \& Eisa, 2012).

Measurement Inc. acquired the rights of PEG in 2002 and continued to develop it. The modified PEG analyzes the training essays and calculates more than 500 features that reflect intrinsic characteristics of writing, such as fluency, diction, grammar, and construction. Once the features have been calculated, the PEG uses them to build statistical and linguistic models for the accurate prediction of essay scores ("Home | Measurement Incorporated," n.d.).

\section{Intelligent Essay Assessor ${ }^{\mathrm{TM}}$ (IEA)}

IEA was developed by Landauer et al. in 1997. IEA uses a statistical combination of several measures to produce an overall score. It relies on using Latent Semantic Analysis (LSA); a machine-learning model of human understanding of the text that depends on the training and calibration methods of the model and the ways it is used tutorially (Dikli, 2006; Foltz, Gilliam, \& Kendall, 2003; Refaat et al., 2012).

IEA can handle students' innovative answers by using a mix of scored essays and the domain content text in the training stage. It also spots plagiarism and provides feedback (Dikli, 2006; Landauer, 2004). It uses a procedure for assigning scores in a process that begins with comparing essays to each other in a set. LSA examines the extremely similar essays. Irrespective of the replacement of paraphrasing, synonym, or reorganization of sentences, the two essays will be similar LSA. Plagiarism is an essential feature to overcome academic dishonesty, which is difficult to be detected by human-raters, especially in the case of grading a large number of essays (Dikli, 2006; Landauer, 2004). (Figure 1) represents IEA architecture (Landauer, 2004). IEA requires smaller numbers of pre-scored essays for training. On the contrary of other AES systems, IEA requires only 100 pre-scored training essays per each prompt vs. 300-500 on other systems (Dikli, 2006).

Landauer et al. in 2003 used IEA to score more than 800 students' answers in middle school. The results showed a 0.90 correlation value between IEA and the human-raters. He explained the high correlation value due to several reasons including that human-raters could not compare each essay to each other for the 800 students while IEA can do so (Dikli, 2006; Landauer, 2004). E-rater ${ }^{\circledR}$ 
136 Educational Testing Services (ETS) developed E-rater in 1998 to estimate the quality of essays 137 in various assessments. It relies on using a combination of statistical and NLP techniques to 138 extract linguistic features (such as grammar, usage, mechanics, development) from text to start 139 processing, then compares scores with human graded essays (Attali \& Burstein, 2014; Dikli, 140 2006; Ramineni \& Williamson, 2018).

141 The E-rater system is upgraded annually. The current version uses 11 features divided into two areas: writing quality (grammar, usage, mechanics, style, organization, development, word

143 choice, average word length, proper prepositions, and collocation usage), and content or use of prompt-specific vocabulary (Ramineni \& Williamson, 2018).

145 The E-rater scoring model consists of two stages: the model of the training stage, and the model of the evaluation stage. Human scores are used for training and evaluating the E-rater scoring models. The quality of the E-rater models and its effective functioning in an operational environment depend on the nature and quality of the training and evaluation data (Williamson, $\mathrm{Xi}, \&$ Breyer, 2012). The correlation between human assessors and the system ranged from 0.87 to 0.94 (Refaat et al., 2012). Criterion is a web-based scoring and feedback system based on ETS text analysis tools: E-rater ${ }^{\circledR}$ and Critique. As a text analysis tool, Critique integrates a collection of modules that detect faults in usage, grammar, and mechanics, and recognizes discourse and undesirable style elements in writing. It provides immediate holistic scores as well (Crozier \& Kennedy, 1994; Dikli, 2006). Criterion similarly gives personalized diagnostic feedback reports based on the types of assessment instructors give when they comment on students' writings. This component of the Criterion is called an advisory component. It is added to the score, but it does not control it[18]. The types of feedback the advisory component may provide are like the following:

- The text is too brief (a student may write more).

- The essay text does not look like other essays on the topic (the essay is off-topic).

- The essay text is overly repetitive (student may use more synonyms).(Crozier \& Kennedy,

164 IntelliMetric $^{\mathrm{TM}}$

Vantage Learning developed the IntelliMetric systems in 1998. It is considered the first AES system which relies on Artificial Intelligence (AI) to simulate the manual scoring process carried out by human-raters under the traditions of cognitive processing, computational linguistics, and classification (Dikli, 2006; Refaat et al., 2012). Processing (NLP) techniques, and statistical techniques. It uses CogniSearch and Quantum

171 Reasoning technologies that were designed to enable IntelliMetric to understand the natural

172 language to support essay scoring (Dikli, 2006).

173 IntelliMetric uses three steps to score essays as follows:

174 a) First, the training step that provides the system with known scores essays. 
175 b) Second, the validation step examines the scoring model against a smaller set of known scores 176 essays.

177 c) Finally, application to new essays with unknown scores. (Learning, 2000, 2003; Shermis \& 178 Barrera, 2002)

179 IntelliMetric identifies text related characteristics as larger categories called Latent Semantic 180 Dimensions (LSD). (Figure 2) represents the IntelliMetric features model.

181 IntelliMetric scores essays in several languages including English, French, German, Arabic, 182 Hebrew, Portuguese, Spanish, Dutch, Italian, and Japanese (Elliot, 2003). According to Rudner, 183 Garcia, and Welch (L. M. Rudner, Garcia, \& Welch, 2006), the average of the correlations 184 between IntelliMetric and human-raters was 0.83 (Refaat et al., 2012).

185 MY Access!

186 MY Access is a web-based writing assessment system based on the IntelliMetric AES system.

187 The primary aim of this system is to provide immediate scoring and diagnostic feedback for the 188 students' writings in order to motivate them to improve their writing proficiency on the topic 189 (Dikli, 2006).

190 MY Access system contains more than 200 prompts that assist in an immediate analysis of the 191

192

193

194 essay. It can provide personalized Spanish and Chinese feedback on several genres of writing such as narrative, persuasive, and informative essays. Moreover, it provides multilevel feedback - developing, proficient, and advanced - as well (Dikli, 2006; Learning, 2003).

Bayesian Essay Test Scoring System ${ }^{\mathrm{TM}}$ (BETSY)

195

196 BETSY classifies the text based on trained material. It has been developed in 2002 by Lawrence Rudner at the College Park of the University of Maryland with funds from the U.S. Department 197

198 of Education (Valenti et al., 2017). It has been designed to automate essay scoring, but can be applied to any text classification task (Taylor, 2005).

199 BETSY needs to be trained on a huge number (1000 texts) of human classified essays to learn

200 how to classify new essays. The goal of the system is to determine the most likely classification 201 of an essay to a set of groups (Pass-Fail) and (Advanced - Proficient - Basic - Below Basic) 202 (Dikli, 2006; Valenti et al., 2017). It learns how to classify a new document through the 203 following steps:

204 The first-word training step is concerned with the training of words, evaluating database 205 statistics, eliminating infrequent words, and determining stop words.

206 The second-word pairs training step is concerned with evaluating database statistics, eliminating 207 infrequent word-pairs, maybe scoring the training set, and trimming misclassified training sets. 208 Finally, BETSY can be applied to a set of experimental texts to identify the classification 209 precision for several new texts or a single text. (Dikli, 2006)

210 BETSY has achieved accuracy of over $80 \%$, when trained with 462 essays, and tested with 80 211 essays (L. M. Rudner \& Liang, 2002).

212

213 Automatic Featuring AES Systems 214 Automatic Text Scoring Using Neural Networks 
215 Alikaniotis, Yannakoudakis, and Rei introduced in 2016 a deep neural network model capable of 216 learning features automatically to score essays. This model has introduced a novel method to 217 identify the more discriminative regions of the text using: 1) a Score-Specific Word Embedding 218 (SSWE) to represent words and 2) a two-layer Bidirectional Long-Short-Term Memory (LSTM) 219 network to learn essay representations. (Alikaniotis, Yannakoudakis, \& Rei, 2016; Taghipour \& $220 \mathrm{Ng}, 2016)$.

221 Alikaniotis and his colleagues have extended the $C \& W$ Embeddings model into the Augmented $222 C \& W$ model to capture, not only the local linguistic environment of each word, but also how 223 each word subsidizes to the overall score of an essay. In order to capture SSWEs. A further linear 224 unit has been added in the output layer of the previous model which performs linear regression, 225 predicting the essay score (Alikaniotis et al., 2016). (Figure 3) shows the architectures of two 226 models, A) Original C\&W model and B) Augmented C\&W model. (Figure 4) shows the 227 example of A) standard neural embeddings to B) SSWE word embeddings.

228 SSWEs obtained by their model used to derive continuous representations for each essay. Each

229

230

231

232

233

234

235

236

237

238

239

240

241

242

243

244

245

246

247

248

249

250

251

252 essay is identified as a sequence of tokens. The uni- and bi-directional LSTMs have been efficiently used for embedding long sequences (Alikaniotis et al., 2016).

They used the Kaggle's ASAP ${ }^{1}$ contest dataset. It consists of 12.976 essays, with average length 150 -to-550 words per essay, each double marked (Cohen's $=0.86$ ). The essays presented eight different prompts, each with distinct marking criteria and score range.

Results showed that SSWE and the LSTM approach, without any prior knowledge of the language grammar or the text domain, was able to mark the essays in a very human-like way, beating other state-of-the-art systems. Furthermore, while tuning the models' hyperparameters on a separate validation set (Alikaniotis et al., 2016), they did not perform any further preprocessing of the text other than simple tokenization. Also, it outperforms the traditional SVM model by combining SSWE and LSTM. On the contrary, LSTM alone did not give significant more accuracies compared to SVM.

According to Alikaniotis, Yannakoudakis, and Rei (Alikaniotis et al., 2016), the combination of SSWE with the two-layer bi-directional LSTM had the highest correlation value on the test set averaged 0.91 (Spearman) and 0.96 (Pearson).

\section{A Neural Network Approach to Automated Essay Scoring}

Taghipour and H. T. Ng developed in 2016 a Recurrent Neural Networks (RNNs) approach which automatically learns the relation between an essay and its grade. Since the system is based on RNNs, it can use non-linear neural layers to identify complex patterns in the data and learn them, and encode all the information required for essay evaluation and scoring (Taghipour $\& \mathrm{Ng}$, 2016).

The designed model architecture can be presented in five layers as follow:

a) The Lookup Table Layer; which builds $d_{\mathrm{LT}}$ dimensional space containing each word projection.

${ }^{1}$ https://www.kaggle.com/c/asap-aes/data 
253 b) The Convolution Layer; which extracts feature vectors from n-grams. It can possibly capture

254

255

256

257

258

259

260

261

262

263

264

265

266

267

268

269

270

271

272

273

274

275

276

277

278

279

280

281

282

283

284

285

286

287

288

289

290 system.

c) The Recurrent Layer; which processes the input to generate a representation for the given essay.

d) The Mean over Time; which aggregates the variable number of inputs into a fixed length vector.

e) The Linear Layer with Sigmoid Activation; which maps the generated output vector from the mean-over-time layer to a scalar value. (Taghipour \& Ng, 2016)

Taghipour and his colleagues have used the Kaggle's ASAP contest dataset. They distributed the data set into $60 \%$ training set, $20 \%$ a development set, and $20 \%$ a testing set. They used Quadratic Weighted Kappa (QWK) as an evaluation metric. For evaluating the performance of the system, they compared it to an available open source AES system called the 'Enhanced AI Scoring Engine' (EASE) ${ }^{2}$. To identify the best model, they performed several experiments like Convolutional vs. Recurrent Neural Network, basic RNN vs. Gated Recurrent Units (GRU) vs. LSTM, unidirectional vs. Bidirectional LSTM, and using with vs. without mean-over-time layer (Taghipour \& Ng, 2016).

The results showed multiple observations according to (Taghipour \& Ng, 2016), summarized as follows:

a) RNN failed to get accurate results as LSTM or GRU and the other models outperformed it. This was possibly due to the relatively long sequences of words in writing.

b) The neural network performance was significantly affected with the absence of the mean over-time layer. As a result, it did not learn the task in an exceedingly proper manner.

c) The best model was the combination of ten instances of LSTM models with ten instances of CNN models. The new model outperformed the baseline EASE system by $5.6 \%$ and with averaged QWK value 0.76 .

\section{Automatic Features for Essay Scoring - An Empirical Study}

Dong and Zhang provided in 2016 an empirical study to examine a neural network method to learn syntactic and semantic characteristics automatically for AES, without the need for external pre-processing. They built a hierarchical Convolutional Neural Network (CNN) structure with two levels in order to model sentences separately (Dasgupta, Naskar, Saha, \& Dey, 2018; Dong \& Zhang, 2016).

Dong and his colleague built a model with two parts, summarized as follows:

a) Word Representations: A word embedding is used but does not rely on POS-tagging or other pre-processing.

b) CNN Model: They took essay scoring as a regression task and employed a two-layer CNN model, in which one Convolutional layer is used to extract sentences representations, and the other is stacked on sentence vectors to learn essays representations.

2 https://github.com/edx/ease 
291 The dataset that they employed in experiments is the Kaggle's ASAP contest dataset. The 292 settings of data preparation followed the one that Phandi, Chai, and Ng used (Phandi, Chai, \& $293 \mathrm{Ng}, 2015)$. For domain adaptation (cross-domain) experiments, they followed Phandi, Chai, and $294 \mathrm{Ng}$ (Phandi et al., 2015), by picking four pairs of essay prompts, namely, $1 \rightarrow 2,3 \rightarrow 4,5 \rightarrow 6$ and $2957 \rightarrow 8$, where $1 \rightarrow 2$ denotes prompt one as source domain and prompt 2 as target domain. They 296 used quadratic weighted Kappa (QWK) as the evaluation metric.

297 In order to evaluate the performance of the system, they compared it to EASE system (an open 298 source AES available for public) with its both models Bayesian Linear Ridge Regression 299 (BLRR) and Support Vector Regression (SVR).

300 The Empirical results showed that the two-layer Convolutional Neural Network (CNN) 301 outperformed other baselines (e.g., Bayesian Linear Ridge Regression) on both in-domain and 302 domain adaptation experiments on the Kaggle's ASAP contest dataset. So, the neural features 303 learned by CNN were very effective in essay marking, handling more high-level and abstract 304 information compared to manual feature templates. In domain average, QWK value was 0.73 vs. 3050.75 for human rater (Dong \& Zhang, 2016).

Augmenting Textual Qualitative Features in Deep Convolution Recurrent Neural Network

\section{for Automatic Essay Scoring}

In 2018, Dasgupta et al. proposed a Qualitatively enhanced Deep Convolution Recurrent Neural Network architecture to score essays automatically. The model considers both word- and sentence-level representations. Using a Hierarchical CNN connected with a Bidirectional LSTM model they were able to consider linguistic, psychological and cognitive feature embeddings within a text (Dasgupta et al., 2018). The designed model architecture for the linguistically informed Convolution RNN can be presented in five layers as follow:

a) Generating Embeddings Layer: The primary function is constructing previously trained sentence vectors. Sentence vectors extracted from every input essay are appended with the formed vector from the linguistic features determined for that sentence.

b) Convolution Layer: For a given sequence of vectors with $\mathrm{K}$ windows, this layer function is to apply linear transformation for all these $\mathrm{K}$ windows. This layer is fed by each of the generated word embeddings from the previous layer.

c) Long Short-Term Memory Layer: The main function of this layer is to examine the future and past sequence context by connecting Bidirectional LSTMs (Bi-LSTM) networks.

d) Activation layer: The main function of this layer is to obtain the intermediate hidden layers from the Bi-LSTM layer $h_{1}, h_{2}, \ldots, h_{T}$, and in order to calculate the weights of sentence contribution to the final essay's score (quality of essay). They used an attention pooling layer over sentence representations.

e) The Sigmoid Activation Function Layer: The main function of this layer is to perform a linear transformation of the input vector that converts it to a scalar value (continuous).

330 (Figure 5) represents the proposed linguistically informed Convolution Recurrent Neural 331 Network architecture. 
332 Dasgupta and his colleagues employed in their experiments the Kaggle's ASAP contest dataset.

333 They have done 7 folds using cross validation technique to assess their models. Every fold is

334

335

336

337

338

339

340

341

342

343

344

345

346

347

348

349

350

351

352

353

354

355

356

357

358

359

360

361

362

363

364

365

366

367

368

369

370

371

372 distributed as follows; training set which represents $80 \%$ of the data, development set represented by $10 \%$, and the rest $10 \%$ as the test set. They used quadratic weighted Kappa (QWK) as the evaluation metric.

The results showed that, in terms of all these parameters, the Qualitatively Enhanced Deep Convolution LSTM (Qe-C-LSTM) system performed better than the existing, LSTM, Bi-LSTM and EASE models. It achieved a Pearson's and Spearman's correlation of 0.94 and 0.97 respectively as compared to that of 0.91 and 0.96 in (Alikaniotis et al., 2016). They also accomplished an RMSE score of 2.09. They computed a pairwise Cohen's $k$ value of 0.97 as well (Dasgupta et al., 2018).

\section{Summary and Discussion}

Over the past four decades, there have been several studies that examined the approaches of applying computer technologies on scoring essay questions. Recently, computer technologies have been able to assess the quality of writing using AES technology. Many attempts have taken place in developing AES systems in the past years (Dikli, 2006).

The AES systems do not assess the intrinsic qualities of an essay directly as human-raters do, but they utilize the correlation coefficients of the intrinsic qualities to predict the score to be assigned to an essay. The performance of these systems is evaluated based on the comparison of the scores assigned to a set of essays scored by expert humans.

The AES systems have many strengths mainly in reducing labor-intensive marking activities, overcoming time, cost, and improving the reliability of writing tasks. Besides, they ensure a consistent application of marking criteria, therefore facilitating equity in scoring. However, there is a substantial manual effort involved in reaching these results on different domains, genres, prompts, and so forth. Moreover, the linguistic features intended to capture the aspects of writing to be assessed are hand-selected and tuned for specific domains. In order to perform well on different data, separate models with distinct feature sets are typically tuned (Burstein, 2003; Dikli, 2006; Hamp-Lyons, 2001; L. Rudner \& Gagne, 2001; L. M. Rudner \& Liang, 2002). Despite its weaknesses, the AES systems continue to attract the attention of public schools, universities, testing agencies, researchers and educators. (Dikli, 2006).

The AES systems described in this paper under the first category are based on handcrafted features and, usually, rely on regression methods. They employ several methods to obtain the scores. While E-rater and IntelliMetric use NLP techniques, the IEA system utilizes LSA. Moreover, PEG utilizes proxy measures (proxes), and BETSYTM uses Bayesian procedures to evaluate the quality of a text.

While E-rater, IntelliMetric, and BETSY evaluate style and semantic content of essays, PEG only evaluates style and ignores the semantic aspect of essays. Furthermore, IEA is exclusively concerned with semantic content. Unlike PEG, E-rater, IntelliMetric, and IEA need smaller numbers of pre-scored essays for training in contrast with BETSY which needs a huge number of training pre-scored essays.

Peer) Comput. Sci. reviewing PDF | (CS-2019:04:37187:1:0:NEW 13 Jun 2019) 
373 The systems in the first category have high correlations with human-raters. While PEG, E-rater, 374 IEA, and BETSY evaluate only English language essay responses, IntelliMetric evaluates essay 375 responses in multiple languages.

376 Contrary to PEG, IEA, and BETSY, E-rater, and IntelliMetric have instructional or immediate

377 feedback applications (i.e., Criterion and MY Access!). Instructional-based AES systems have 378 worked hard to provide formative assessments by allowing students to save their writing drafts 379 on the system. Thus, students can review their writings as of the formative feedback received 380 from either the system or the teacher. The recent version of MY Access! (6.0) provides online 381 portfolios and peer review.

382 The drawbacks of this category may include the following: a) feature engineering can be time-

383

384

385

386

387

388

389

390

391

392

393

394

395

396

397

398

399

400

401

402

403

404

405

406

407

408

409

410

411

412 consuming, since features need to be carefully handcrafted and selected to fit the appropriate model, and b) such systems are sparse and instantiated by discrete pattern-matching.

AES systems described in this paper under the second category are usually based on neural networks. Neural Networking approaches, especially Deep Learning techniques, have been shown to be capable of inducing dense syntactic and semantic features automatically, applying them to text analysis and classification problems including AES systems (Alikaniotis et al., 2016; Dong \& Zhang, 2016; Taghipour \& Ng, 2016), and giving better results with regards to the statistical models used in the handcrafted features (Dong \& Zhang, 2016).

Recent advances in Deep Learning have shown that neural approaches to AES achieve state-ofthe-art results (Alikaniotis et al., 2016; Taghipour \& Ng, 2016) with the additional advantage of utilizing features that are automatically learned from the data. In order to facilitate interpretability of neural models, a number of visualization techniques have been proposed to identify textual (superficial) features that contribute to model performance [7].

While Alikaniotis and his colleagues (2016) employed a two-layer Bidirectional LSTM combined with the SSWE for essay scoring tasks, Taghipour and Ng (2016) adopted the LSTM model and combined it with CNN. Dong and Zhang (2016) developed a two-layer CNN, and Dasgupta and his colleagues (2018) proposed a Qualitatively Enhanced Deep Convolution LSTM. Unlike Alikaniotis and his colleagues (2016), Taghipour and Ng (2016), Dong and Zhang (2016), Dasgupta and his colleagues (2018) were interested in word-level and sentencelevel representations as well as linguistic, cognitive and psychological feature embeddings. All linguistic and qualitative features were figured off-line and then entered in the Deep Learning architecture.

Although Deep Learning-based approaches have achieved better performance than the previous approaches, the performance may not be better using the complex linguistic and cognitive characteristics, which are very important in modeling such essays. See (Table 1) for the comparison of AES systems.

In general, there are three primary challenges to AES systems. First, they are not able to assess essays as human-raters do because they do what they have been programmed to do (Page, 2003). They eliminate the human element in writing assessment and lack the sense of the rater as a person (Hamp-Lyons, 2001). This shortcoming was somehow overcome by obtaining high 
413 correlations between the computer and human-raters (Page, 2003) although this is still a

414 challenge.

415 The second challenge is whether the computer can be fooled by students or not (Dikli, 2006). It

416 is likely to "trick" the system by writing a longer essay to obtain higher score for example

417 (Kukich, 2000). Studies, such as the GRE study in 2001, examined whether a computer could be

418 deceived and assign a lower or higher score to an essay than it should deserve or not. The results

419 revealed that it might reward a poor essay (Dikli, 2006). The developers of AES systems have

420 been utilizing algorithms to detect students who try to cheat.

421 Although automatic learning AES systems based on Neural Networks algorithms, the

422 handcrafted AES systems transcend automatic learning systems in one important feature.

423 Handcrafted systems are highly related to the scoring rubrics that have been designed as a

424 criterion for assessing a specific essay and human-raters use these rubrics to score essays a well.

425 The objectivity of human-raters is measured by their commitment to the scoring rubrics. On the

426 contrary, automatic learning systems extract the scoring criteria using machine learning and

427 neural networks, which may include some factors that are not part of the scoring rubric, and,

428 hence, is reminiscent of raters' subjectivity (i.e., mode, nature of a rater's character, etc.)

429 Considering this point, handcrafted AES systems may be considered as more objective and fairer 430 to students from the viewpoint of educational assessment.

431 The third challenge is measuring the creativity of human writing. Accessing the creativity of 432 ideas and propositions and evaluating their practicality are still a pending challenge to both 433 categories of AES systems which still needs further research.

434

\section{References}

436

437

438

439

440

441

442

443

444

445

446

447

448

449

450

451

452

Alikaniotis, D., Yannakoudakis, H., \& Rei, M. (2016). Automatic Text Scoring Using Neural Networks. https://doi.org/10.18653/v1/P16-1068

Attali, Y., \& Burstein, J. (2014). Automated Essay Scoring With E-Rater ${ }^{\circledR}$ V.2.0. ETS Research Report Series, 2004(2), i-21. https://doi.org/10.1002/j.2333-8504.2004.tb01972.x

Burstein, J. (2003). The e-rater Scoring Engine: Automated Essay Scoring with Natural Language Processing, 107-115. Retrieved from http://books.google.com/books?id=JIR6ihd7ZA4C\&printsec=frontcover $\# \mathrm{v}=$ onepage\&q\&f= false

Crozier, W. W., \& Kennedy, G. J. A. (1994). Marine exploitation of Atlantic salmon (Salmo salar L.) from the River Bush, Northern Ireland. In Fisheries Research (Vol. 19, pp. 141155). https://doi.org/10.1016/0165-7836(94)90020-5

Dasgupta, T., Naskar, A., Saha, R., \& Dey, L. (2018). Augmenting Textual Qualitative Features in Deep Convolution Recurrent Neural Network for Automatic Essay Scoring. Aclweb.Org, 93-102. Retrieved from http://aclweb.org/anthology/W18-3713

Dikli, S. (2006). An Overview of Automated Scoring of Essays. The Journal Of Technology, Learning, and Assessment, 5(1), 1-36.

Dong, F., \& Zhang, Y. (2016). Automatic Features for Essay Scoring - An Empirical Study. In 
453

454

455

456

457

458

459

460

461

462

463

464

465

466

467

468

469

470

471

472

473

474

475

476

477

478

479

480

481

482

483

484

485

486

487

488

489

490

491

492

\section{Proceedings of the 2016 Conference on Empirical Methods in Natural Language}

Processing. (pp. 1072-1077). https://doi.org/10.18653/v1/d16-1115

Elliot, S. (2003). IntelliMetric: From here to validity. Automated Essay Scoring: A CrossDisciplinary Perspective, 71-86. Retrieved from https://ci.nii.ac.jp/naid/10025900425/

Farag, Y., Yannakoudakis, H., \& Briscoe, T. (2018). Neural Automated Essay Scoring and Coherence Modeling for Adversarially Crafted Input, 263-271. https://doi.org/10.18653/v1/N18-1024

Foltz, P. W., Gilliam, S., \& Kendall, S. (2003). Supporting Content-Based Feedback in On-Line Writing Evaluation with LSA. Interactive Learning Environments, 8(2), 111-127. https://doi.org/10.1076/1049-4820(200008)8:2;1-b;ft111

Hamp-Lyons, L. (2001). Fourth generation writing assessement. On Second Language Writing, $117,117-128$.

Home | Measurement Incorporated. (n.d.). Retrieved February 5, 2019, from http://www.measurementinc.com/

Isaacs, T. (2013). Key Concepts in Educational Assessment [electronic resource]. Sage.

Kukich, K. (2000). Beyond automated essay scoring, the debate on automated essay grading. IEEE Intelligent Systems, 15(5), 22-27.

Landauer, T. K. (2004). Automatic Essay Assessment. Assessment in Education: Principles, Policy \& Practice, 10(3), 295-308. https://doi.org/10.1080/0969594032000148154

Learning, V. (2000). A true score study of IntelliMetric accuracy for holistic and dimensional scoring of college entry-level writing program (RB-407). Newtown, PA: Vantage Learning.

Learning, V. (2003). A true score study of 11th grade student writing responses using IntelliMetric Version 9.0 (RB-786). Newtown, PA: Vantage Learning, 1.

Nitko, A. J., \& Brookhart, S. M. (2007). Educational Assessment of Students, 5th edition. Pearson Merrill Prentice Hall.

Page, E. B. (1994). Computer grading of student prose, using modern concepts and software. Journal of Experimental Education, 62(2), 127-142. https://doi.org/10.1080/00220973.1994.9943835

Page, E. B. (2003). Project essay grade: PEG. Automated Essay Scoring: A Cross-Disciplinary Perspective.

Peng, X., Ke, D., \& Xu, B. (2012). Automated Essay Scoring Based on Finite State Transducer: towards ASR Transcription of Oral English Speech. Jeju, Republic of Korea, (July), 50-59.

Phandi, P., Chai, K. M. A., \& Ng, H. T. (2015). Flexible Domain Adaptation for Automated Essay Scoring Using Correlated Linear Regression. Wiley, 431-439. https://doi.org/10.18653/v1/d15-1049

Ramineni, C., \& Williamson, D. (2018). Understanding Mean Score Differences Between the erater ${ }^{\circledR}$ Automated Scoring Engine and Humans for Demographically Based Groups in the GRE ${ }^{\circledR}$ General Test. ETS Research Report Series. https://doi.org/10.1109/ISIE.1997.648935

Refaat, M. M., Ewees, A. A., \& Eisa, M. M. (2012). Automated Assessment of Students'Arabic 
493

494

495

496

497

498

499

500

501

502

503

504

505

506

507

508

509

510

511

512

513

514

515

516

517

518

519

520

521

522

523

524

525

526

Free-Text Answers. International Journal of Intelligent Computing And Information Science, 12(1), 213-222. Retrieved from

https://www.researchgate.net/profile/Ahmed_Ewees/publication/236019860_AUTOMATE D_ASSESSMENT_OF_STUDENTS\%27_ARABIC_FREETEXT_ANSWERS/links/5a9afae8aca2721e3f3017d4/AUTOMATED-ASSESSMENT-OFSTUDENTS-ARABIC-FREE-TEXT-ANSWERS.pdf

Rudner, L., \& Gagne, P. (2001). An Overview of Three Approaches to Scoring Written Essays by Computer. ERIC Digest.

Rudner, L. M., Garcia, V., \& Welch, C. (2006). An Evaluation of the IntelliMetricSM Essay Scoring System. The Journal of Technology, Learning and Assessment, 4(4), 3-20.

Retrieved from https://ejournals.bc.edu/ojs/index.php/jtla/article/view/1651/1493

Rudner, L. M., \& Liang, T. (2002). Automated Essay Scoring Using Bayes' Theorem. The Journal of Technology, Learning, and Assessment, 1(2), 1-21. Retrieved from http://napoleon.bc.edu/ojs/index.php/jtla/article/view/1668

Shermis, M. D., \& Barrera, F. D. (2002). Exit assessments evaluating writing ability through automated essay scoring. Annual Meeting of the American Educational Research Association, New Orleans, LA, 1-30. Retrieved from http://search.ebscohost.com/login.aspx?direct=true\&db=eric\&AN=ED464950\&site=ehostlive

Stecher, B. M., Rahn, M., Ruby, A., Alt, M., Robyn, A., \& Ward, B. (1997). Types of Assessment. Using Alternative Assessments in Vocational Education.

Taghipour, K., \& Ng, H. T. (2016). A Neural Approach to Automated Essay Scoring. In Proceedings of the 2016 Conference on Empirical Methods in Natural Language Processing (pp. 1882-1891). https://doi.org/10.18653/v1/d16-1193

Taylor, A. R. (2005). A Future in the Process of Arrival : Using Computer Technologies for the Assessment of Student Learning. Retrieved from http://site.ebrary.com/lib/uwo/docDetail.action?docID=10276954

Valenti, S., Neri, F., \& Cucchiarelli, A. (2017). An Overview of Current Research on Automated Essay Grading. Journal of Information Technology Education: Research, 2, 319-330. https://doi.org/10.28945/331

Williamson, D. M., Xi, X., \& Breyer, F. J. (2012). A Framework for Evaluation and Use of Automated Scoring. Educational Measurement: Issues and Practice, 31(1), 2-13. https://doi.org/10.1111/j.1745-3992.2011.00223.x 


\section{Table 1 (on next page)}

The comparison of AES systems 


\begin{tabular}{|c|c|c|c|c|c|c|c|}
\hline AES/Parameter & Vendor & $\begin{array}{c}\text { Release } \\
\text { date }\end{array}$ & Primary focus & $\begin{array}{c}\text { Technique(s) } \\
\text { used }\end{array}$ & $\begin{array}{c}\text { Training } \\
\text { data }\end{array}$ & $\begin{array}{c}\text { Feedback } \\
\text { Application }\end{array}$ & $\begin{array}{c}\text { Correlation } \\
\text { with human } \\
\text { raters' scores }\end{array}$ \\
\hline $\mathrm{PEG}^{\mathrm{TM}}$ & Ellis Page & 1966 & Style & Statistical & $\begin{array}{c}\text { Yes } \\
(100-400)\end{array}$ & No & 0.87 \\
\hline IEA $^{\mathrm{TM}}$ & $\begin{array}{l}\text { Landauer, Foltz, } \\
\text { \& Laham }\end{array}$ & 1997 & Content & $\begin{array}{l}\text { LSA (KAT } \\
\text { engine by } \\
\text { PEARSON) }\end{array}$ & $\begin{array}{l}\text { Yes } \\
(\sim 100)\end{array}$ & Yes & 0.90 \\
\hline E-rater ${ }^{\circledR}$ & $\begin{array}{c}\text { ETS } \\
\text { development } \\
\text { team }\end{array}$ & 1998 & Style \& Content & NLP & $\begin{array}{c}\text { Yes } \\
(\sim 400)\end{array}$ & $\begin{array}{c}\text { Yes } \\
\text { (Criterion) }\end{array}$ & $\sim 0.91$ \\
\hline IntelliMetric ${ }^{\mathrm{TM}}$ & $\begin{array}{c}\text { Vantage } \\
\text { Learning }\end{array}$ & 1998 & Style \& Content & NLP & $\begin{array}{c}\text { Yes } \\
(\sim 300)\end{array}$ & $\begin{array}{c}\text { Yes } \\
\text { (MY Access!) }\end{array}$ & $\sim 0.83$ \\
\hline BETSYTM & Rudner & 1998 & Style \& Content & $\begin{array}{l}\text { Bayesian text } \\
\text { classification }\end{array}$ & $\begin{array}{c}\text { Yes } \\
(1000)\end{array}$ & No & $\sim 0.80$ \\
\hline $\begin{array}{l}\text { D. Alikaniotis, } \\
\text { H. } \\
\text { Yannakoudakis, } \\
\text { and M. Rei } \\
\text { (Alikaniotis, } \\
\text { Yannakoudakis, } \\
\text { \& Rei, 2016) }\end{array}$ & $\begin{array}{c}\text { Alikaniotis, } \\
\text { Yannakoudakis, } \\
\text { and Rei }\end{array}$ & 2016 & Style \& Content & $\begin{array}{l}\text { SSWE + Two- } \\
\text { layer Bi-LSTM }\end{array}$ & $\begin{array}{c}\text { Yes } \\
(\sim 8000)\end{array}$ & No & $\begin{array}{c}\sim 0.91 \\
\text { (Spearman) } \\
\sim 0.96 \\
\text { (Pearson) }\end{array}$ \\
\hline $\begin{array}{l}\text { Taghipour and } \\
\mathrm{Ng}(\text { Taghipour } \\
\& \mathrm{Ng}, 2016)\end{array}$ & $\begin{array}{c}\text { Taghipour and } \\
\mathrm{Ng}\end{array}$ & 2016 & Style \& Content & Adopted LSTM & $\begin{array}{c}\text { Yes } \\
(\sim 7786)\end{array}$ & $\mathrm{NO}$ & $\begin{array}{c}\text { QWK for } \\
\text { LSTM } \sim 0.761\end{array}$ \\
\hline $\begin{array}{l}\text { Dong and Zhang } \\
\text { (Dong \& Zhang, } \\
\text { 2016) }\end{array}$ & Dong and Zhang & 2016 & $\begin{array}{c}\text { Syntactic and } \\
\text { semantic } \\
\text { features }\end{array}$ & $\begin{array}{c}\text { Word } \\
\text { embedding and } \\
\text { a two-layer } \\
\text { Convolution } \\
\text { Neural Network }\end{array}$ & $\begin{array}{c}\text { Yes } \\
(\sim 1500 \text { to } \\
\sim 1800)\end{array}$ & $\mathrm{NO}$ & $\begin{array}{c}\text { average kappa } \\
\sim 0.734 \text { versus } \\
0.754 \text { for } \\
\text { human }\end{array}$ \\
\hline $\begin{array}{l}\text { T. Dasgupta, A. } \\
\text { Naskar, L. Dey } \\
\text { and R. Saha } \\
\text { (Dasgupta, } \\
\text { Naskar, Saha, \& } \\
\text { Dey, 2018) }\end{array}$ & $\begin{array}{l}\text { Dasgupta, T., } \\
\text { Naskar, A., Dey, } \\
\text { L., \& Saha, R. }\end{array}$ & 2018 & $\begin{array}{l}\text { Style, Content, } \\
\text { linguistic and } \\
\text { psychological }\end{array}$ & $\begin{array}{c}\text { Deep } \\
\text { Convolution } \\
\text { Recurrent } \\
\text { Neural Network }\end{array}$ & $\begin{array}{c}\text { Yes } \\
(\sim 8000 \text { to } \\
10000)\end{array}$ & $\mathrm{NO}$ & $\begin{array}{l}\text { Pearson's and } \\
\text { Spearman's } \\
\text { correlation of } \\
0.94 \text { and } 0.97 \\
\text { respectively }\end{array}$ \\
\hline
\end{tabular}


Figure 1

The IEA architecture

\section{Customized}

Reader

$\%$ Content

$\%$ Style

$\%$ Mechanics

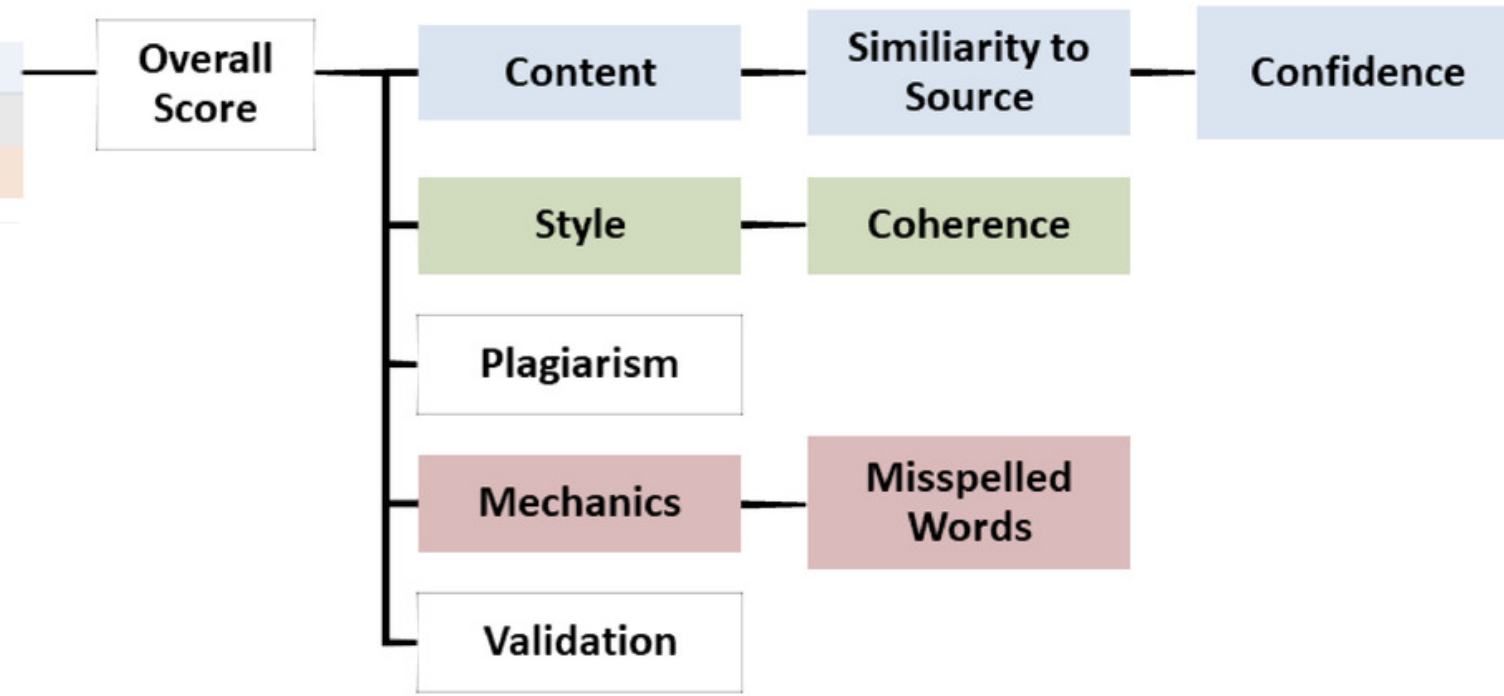


Figure 2

The IntelliMetric features model

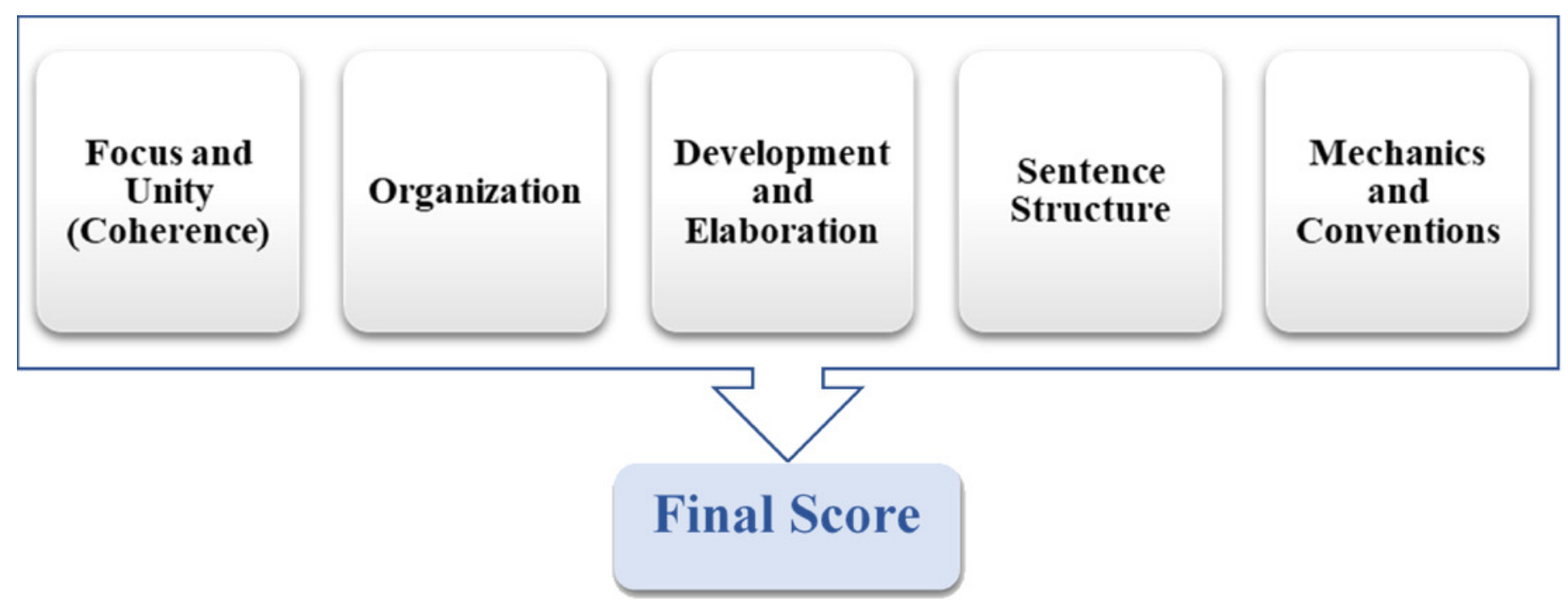


Figure 3

The architectures of two models

(A) Original C\&W model. (B) Augmented C\&W model

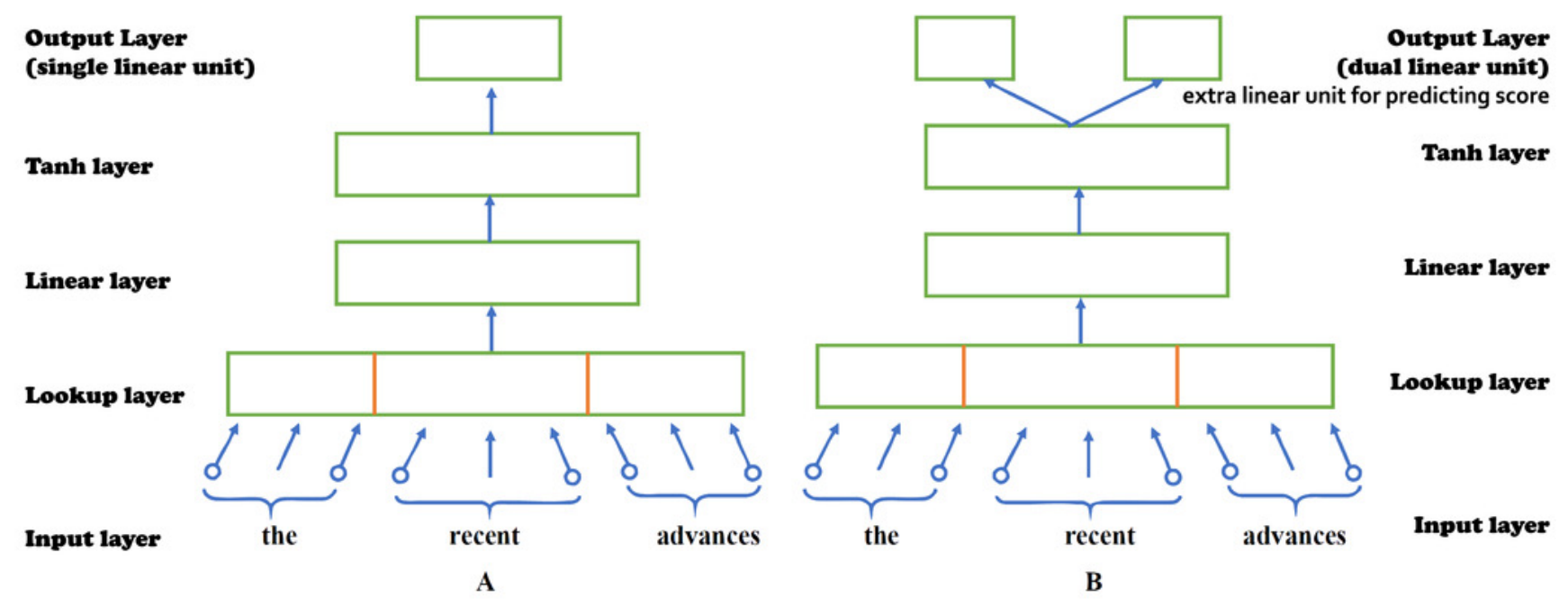


Figure 4

The example of embeddings

(A) standard neural embeddings. (B) SSWE word embeddings
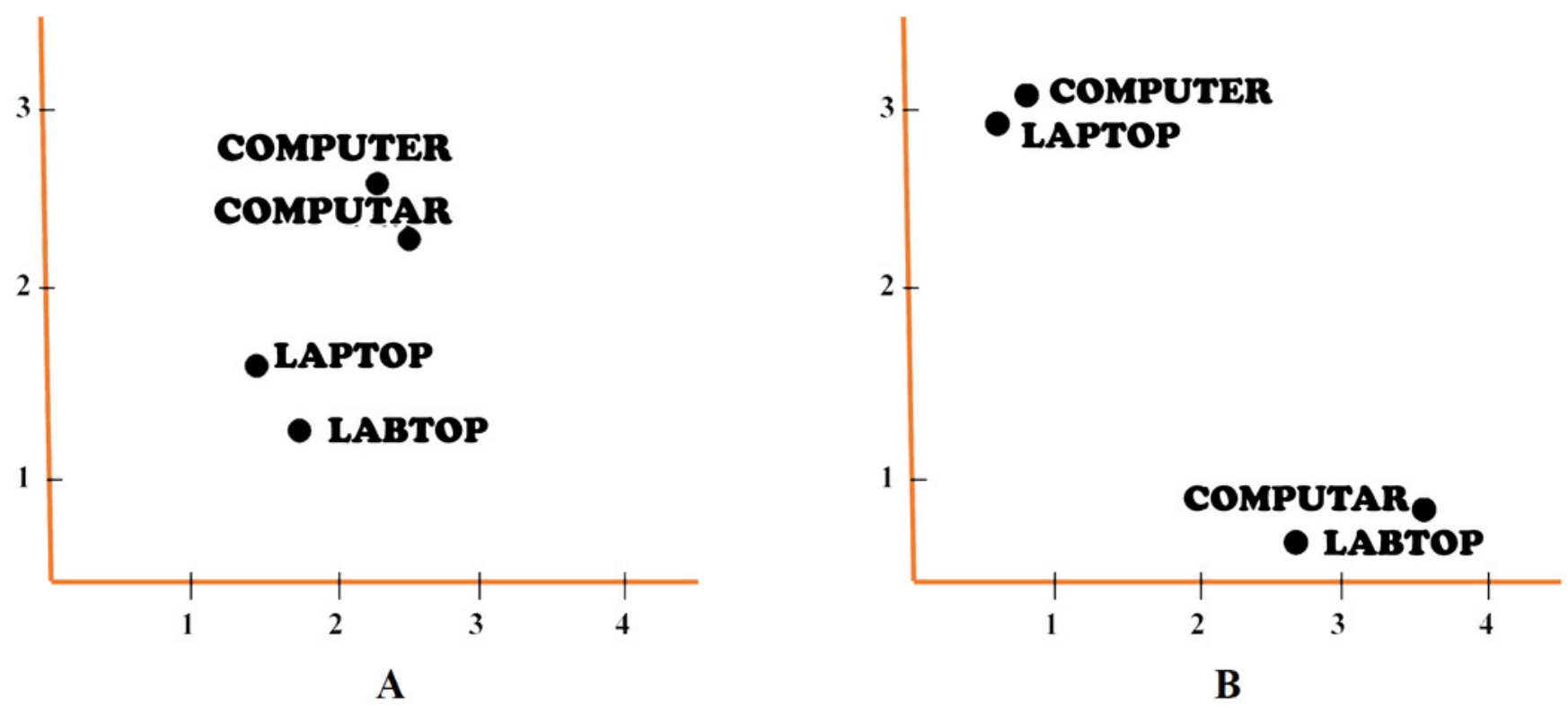
Figure 5

The proposed linguistically informed Convolution Recurrent Neural Network architecture

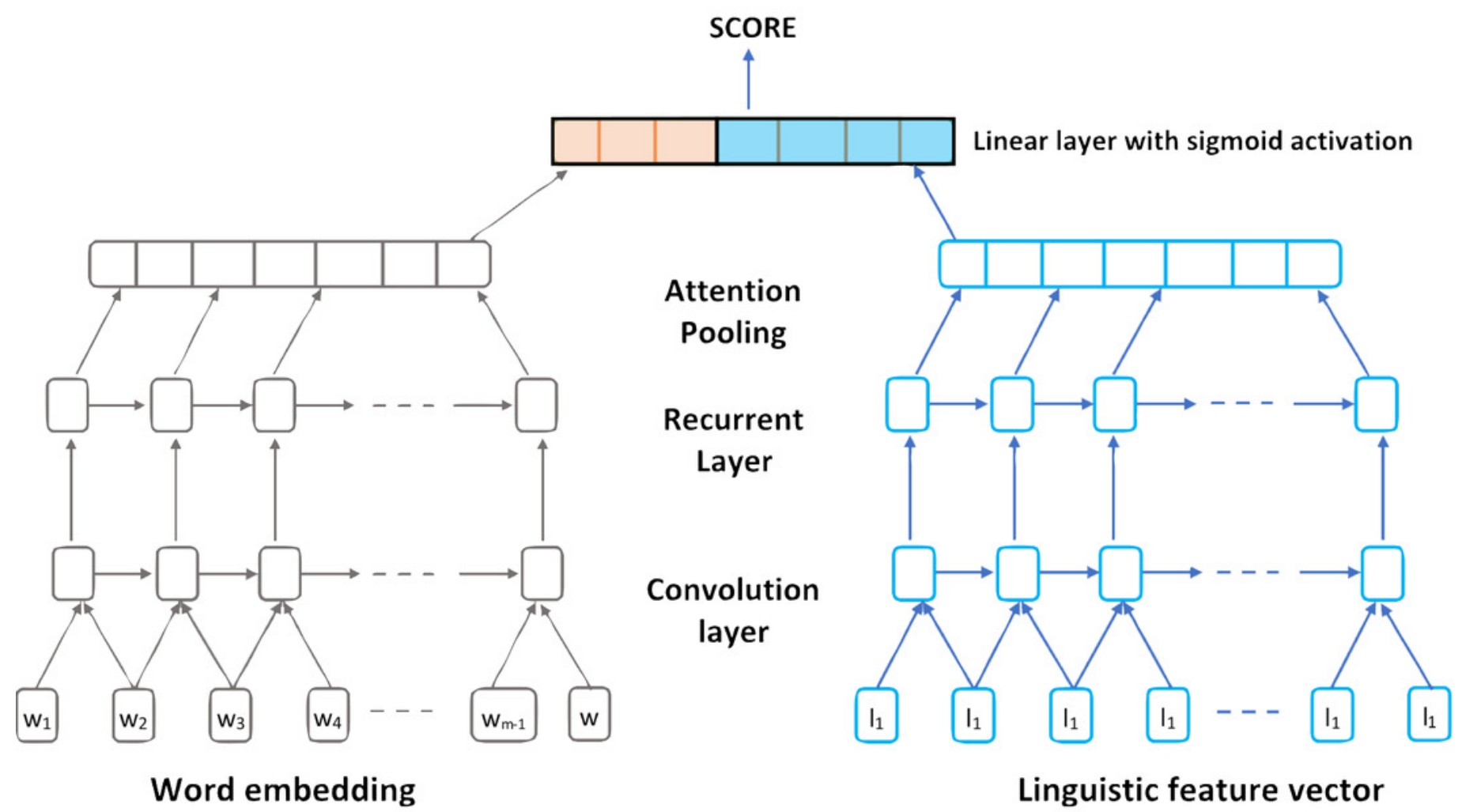

